\title{
EYE CONTROLLED SWITCHING USING CIRCULAR HOUGH TRANSFORM
}

\author{
Sagar Lakhmani \\ Software Engineering Associate, \\ Accenture Services Private Limited, Pune, India \\ Sagar.lakhmani@accenture.com
}

\begin{abstract}
The paper presents hands free interface between electrical appliances or devices. This technology is intended to replace conventional switching devices for the use of disabled. It is a new way to interact with the electrical or electronic devices that we use in our daily life. The paper illustrates how the movement of eye cornea and blinking can be used for switching the devices. The basic Circle Detection algorithm is used to determine the position of eye. Eye blinking is used as triggering action which produces binary output through the parallel port of computer. This output is used for switching the particular device.
\end{abstract}

\section{KEYWORDS}

Eye controlled switching, Circular Hough Transform, Circle detection, Hands-free switching

\section{INTRODUCTION}

The existing appliances used in our daily life such as Fans, Lights, Televisions, and other devices have been used to achieve daily comfort. These electrical appliances cannot be operated by the handicapped persons. In this paper, a computer based input device by human eyes is used for switching of daily use electrical appliances.

The existing Switching methods for interacting with electrical devices are as follows:

1. Voice Based Method, which use user's voice as source input. Voice analysis is used to analyse user's voice and convert into electrical digital signals of ON \& OFF. The weakness of this system is presence of noise. Other voices coming from surrounding may affect the system.

2. Hardware switch, the most common method of interacting with electrical devices.

There have been methods involved for controlling of appliances such as Electric Wheelchair, Computer Mouse, etc using Eye tracking. The existing methods using eye movements as input are as follows:

Sundarapandian et al. (Eds) : CCSEIT, DMDB, ICBB, MoWiN, AIAP - 2014

pp. 09-16, 2014. (C) CS \& IT-CSCP 2014

DOI : $10.5121 /$ csit.2014.4802 
1. Using Infrared Camera, in which the movement of the eye is monitored by high end infrared camera and high precision monitor mounted telescopic cameras. The camera detects the movement of the eye after the calibration process is performed successfully with few training sessions which records reference points given by the system. Once the training is completed, the system uses stored eye positions recorded during the training and current image of the eyes captured by live cameras to plot the expected position using linear interpolation methods. This method required efficient jitter reduction algorithm since the random movement of the user is quite common in practical cases.

2. Using Starburst technique which needs intensive reinforced learning for making the system practically reliable and the cost of implementation is too high. The cameras employed to read the eye movements are highly sophisticated and involve huge investment thereby increasing the cost when production is adopted at a commercial level.

3. Use of KNN classifier to determine various illumination conditions, which is more feasible than lighting compensation processing in real-time implementation. But this is very much dependant on the lighting conditions provided under practical environments and depended on the entire face image acquisition and extracting required features from the frame containing the image of whole face. The extraction of the whole face as the problem domain makes it more complex since the image processing should involve segmentation process to separately isolate the properties of the user's eye.

Many popular eye based appliance handling systems have been developed that generates no feedback when person looks at it. It can be quite distracting to a person when he or she is aware of the gazes and consciously tries to put the efforts to control switching the devices.

\section{PROPOSED METHOD}

The methodology used is based on the output of the implemented circular Hough Transform for circle detection to sense the image of the eyeball using a camera mounted on a headgear and continuously work upon the video frames to detect the position of the cornea. The position of the cornea determines which device is to be switched ON or OFF. The experimental setup has been constructed to contain a head supported structure containing a wireless webcam which captures the close up live video of the eye. An Object Analysis Toolbox available in latest versions of MATLAB enables experimenters to measure the edges and objects in an image. The imfindcircles function of this toolbox is used to detect the position of the cornea.

The following figure [Figure 1] shows the photograph of the hardware device we have used for the experimental purpose. The device consists of a flexible structure which can be worn as a head gear by the user. The tip of the structure is mounted with a simple webcam which captures the image of the eye.

An IR LED is used to illuminate the eyes and this method is technically known as dark-pupil illumination. The main reason for using this technique is the complete absorption of the IR rays into the cornea of the eyes so as to eliminate specular reflection caused when visible light is used instead. This yields results with excellent accuracy by the image processing module we use to detect the centre of the cornea to obtain its coordinates. The inlet shows the captured image on the computer screen. For better illumination, we have adjusted the position of the camera arm such 
that we have minimum blockage of the ambient light present in the laboratory and the shadow of the structure does not fall directly on the cornea.

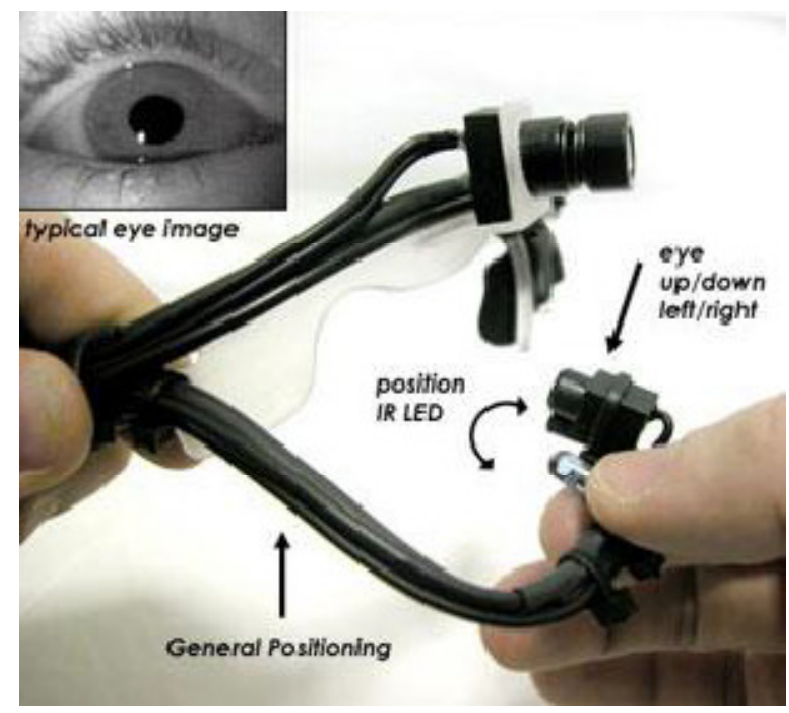

Figure 1: The hardware device used for capturing the live video of the eye.

The calculation is facilitated by assuming an imaginary square grid placed over the input frames and detect the cell in the grid in which the centre of the eye is currently detected by Circular Hough Transform circle detection algorithm. The centre of the cornea can be calculated by monitoring the co-ordinate of the cell. The following captured image illustrates this.

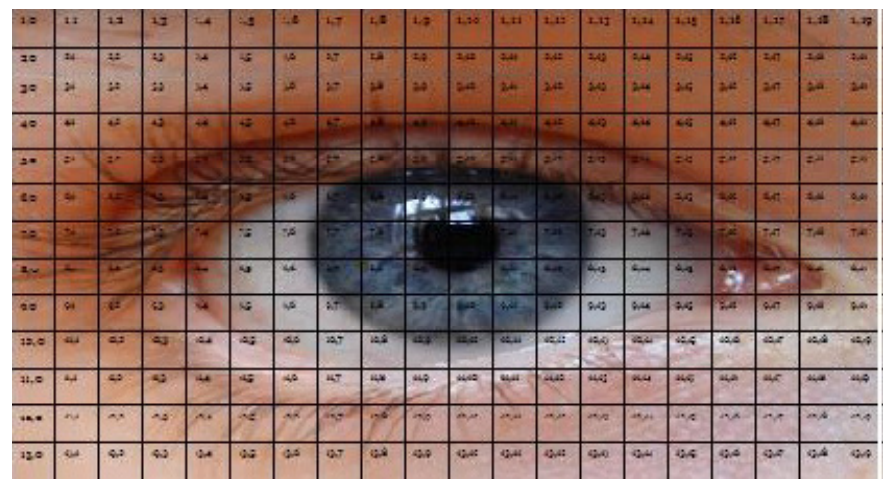

Figure 2: Captured image of the eye is scaled across the virtual grid for processing and device selection.

The grid position of the centre of cornea determines the device to be switched ON or OFF. For every grid we have one device to be operated. For the sake of simplicity, we have considered only the horizontal grids. Vertical grids could also be considered if the number of devices to be operated are more.

There are two control parameters extracted from the input video for switching the appliances. The first parameter is the centre of the cornea which determines the device. The second parameter is the motion of the eyelid i.e. Blinks. The blinks here are used as a trigger for the switch. Once we 
determine the grid position of cornea, a blink can be used to switch that particular device ON or OFF. If the device is ON presently, a blink at the corresponding device grid will switch OFF the device and vice-versa.

The Blinks can be detected using another method of motion detection. For motion detection we have used frame difference algorithm for background subtraction. In this method, using live video we compare two frames of images. If the differences between the two frames is greater than a certain threshold, a motion is detected. This motion detected can be used as a trigger for switch.

The following diagram shows a real time circle detection in an image.

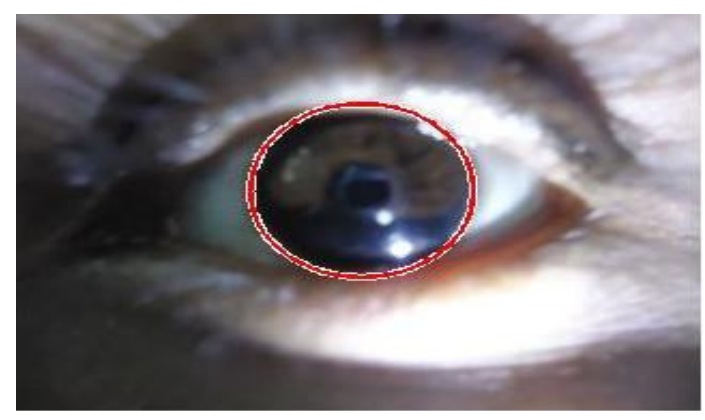

Figure 3: Circle detection using imfindcircles function of MATLAB.

The two parameters i.e. centre co-ordinate of cornea and blink output can be used in a program to give output to the hardware parts through the use of Parallel port of computer.

\section{IMPLEMENTATION \& RESULTS}

The image processing was simulated in MATLAB and the Circular Hough Transform was used to process the image from the live camera to obtain the co-ordinates of the centre of the eye which was then used to decide which device needs to be selected. The motion detection of the eye blink was used to decide whether the device has to be switched ON or OFF. This whole process yielded a output from the parallel port of the computer which is further processed by Transmitter and Receiver blocks of the hardware.

The image processing was simulated in MATLAB and the Circular Hough Transform was used to process the image from the live camera to obtain the co-ordinates of the centre of the eye which was then used to decide which device needs to be selected. The motion detection of the eye blink was used to decide whether the device has to be switched ON or OFF. This whole process yielded a output from the parallel port of the computer which is further processed by Transmitter and Receiver blocks of the hardware.

\subsection{The Transmitter Block}

The Transmitter block takes the output from the parallel port of the computer and processes it with transistorized switch. The output of the transistorized switch is then fed to a DTMF(Dual tone Multiple Frequency) tone generator IC which converts the input to a DTMF output which 
can be transmitted to the remote section where the devices are located. The transmitter block is as shown below:

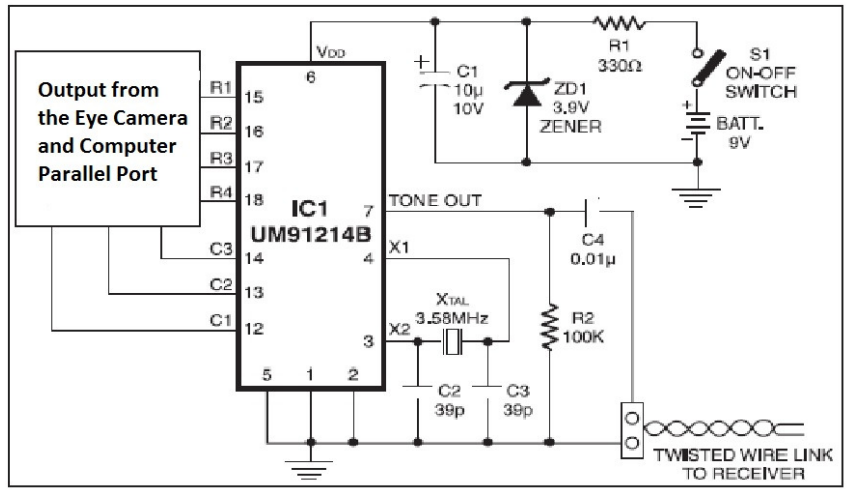

Figure 4: The Transmitter section of the Hardware part.

The transistorized switches used here are Optocouplers (not in the diagram). The optocouplers were used to enable electrical isolation between the computer parallel port and hardware section thereby preventing high voltages to affect the system.

The DTMF tone generator IC UM91214B is used to generate a single signal which can be transmitted to the Receiver section located remotely.

\subsection{The Receiver Block}

The receiver section consists of four parts namely, DTMF decoder, Bit Converter, Flip Flop assembly and relay circuits associated with the END devices to be operated. The circuit diagram of the Receiver block is as shown below:

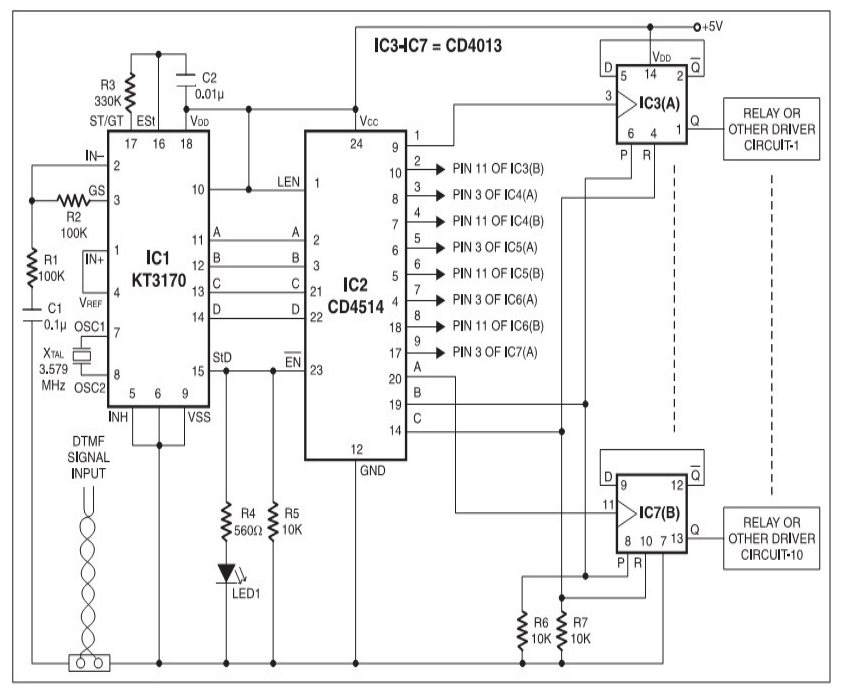

Figure 5: The Receiver section of the Hardware part. 
The DTMF decoder IC KT3170 is used to decode the signal received from the transmitter section. The output of this IC is a 4-bit code containing information about which device is to be operated.

The output of DTMF decoder is fed to a 4-bit to 16-bit converter IC CD4514. The output received from this can be used to operate 16 devices individually. But, for the sake of simplicity we have used only four devices for demonstration.

The output of bit converter IC is then given to flip flop circuits. The output received from the transmitter block and parallel port may last for a very minute time. To hold this output until next triggering stage flip flops are used.

The output of the flip flops is fed to relays associated with the end appliances. The relays are used here as a switch. Since the output from the flip flops is of very low voltage, relays are used to connect the appliances to the power source. The functioning of a relay can be understood by the following diagram:

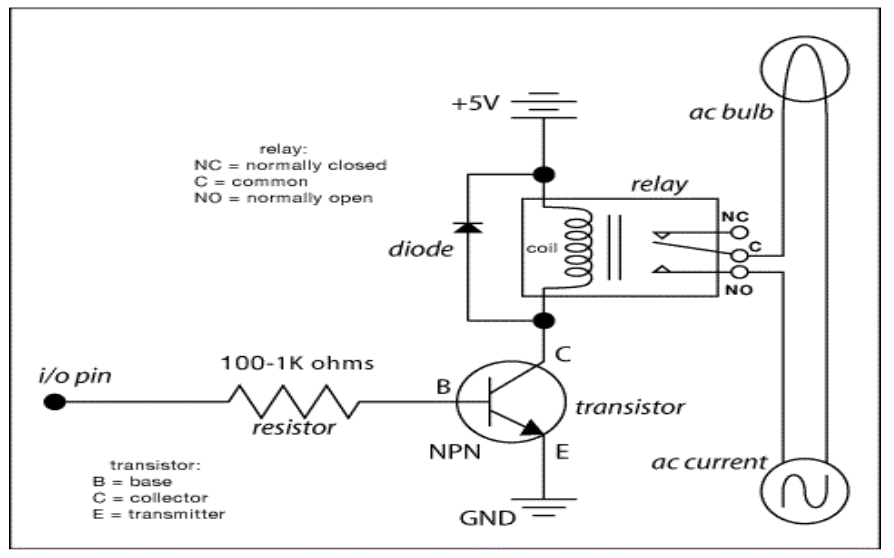

Figure 6: The Relay Assembly

The actual hardware diagram is shown below. It consists of all the above mentioned blocks.

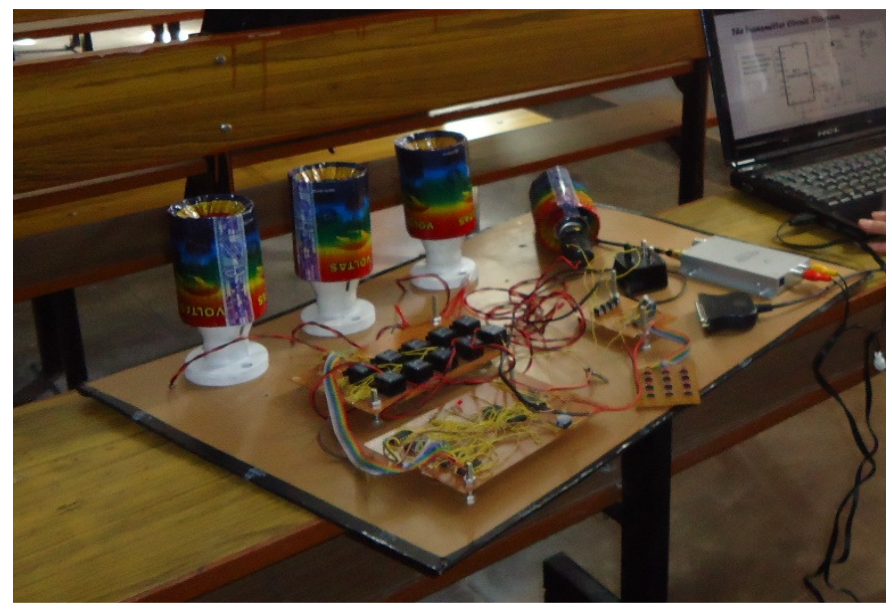

Figure 7: The hardware section. 
For the sake of easiness, we tested this method for four devices which we used as AC bulb. The eye positions were scaled to the grid as shown in the below diagram:

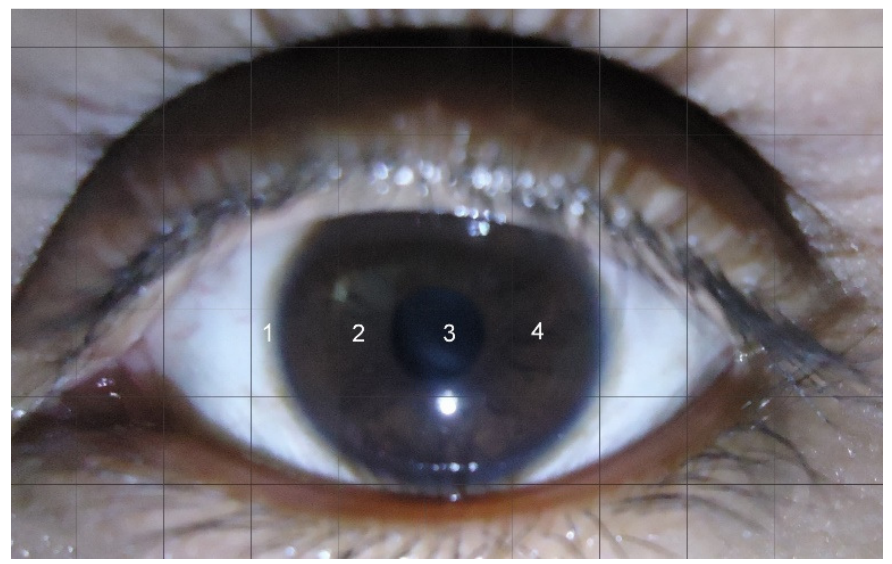

Figure 8: Scaling of eye to 4 grids for demonstration.

If the centre of the eye were to lie in grid 1, and the blinking of eye were done, the bulb 1 will get switched ON. Again if the blinking has been done while keeping the centre of the eye in grid 1, the device would turn off. Similarly, it works for all the four AC bulbs.

\section{CONCLUSION}

The use of standard Circular Hough Transformation in image processing module of the implementation combined with the grid analysis approach was proved to be practically successful and has high potential in future applications of the same for automated wheelchairs for the disabled. The approach has got huge potential once it is optimized in terms of the time complexity with the help of a machine with high end hardware specification. The cursor movement was achieved with a good precision in movement and the totally cost of the experimental hardware used to make the system was well below USD 150 and its commercial implementation can be easily made in affordable price range for the common man.

Moreover, the special features of the proposed method are,

1. It allows user movement. User can move in any direction when Camera is mounted.

2. It does not require any calibration process before using the proposed method.

3. It does not cause fatigue to the eyes as the headgear can be removed when not needed.

\section{FUTURE WORKS}

The implemented system is observed to have huge potential for use in other eye operated devices for the disabled. The future works will be aimed at modifying the approach to make it suitable for use in the eye operated wheelchairs. The wheel chair control system can be controlled using the signals generated by the system working on the discussed logic and can further reduce the cost of the expensive solutions and increase comfort for the users who are in need of the same. 


\section{ACKNOWLEDGMENT}

I would like to express heartfelt gratitude towards my faculty guide Mr. Krishna Mohan Pandey, MATLAB trainer and expert, CETPA infotech Private Limited, Lucknow, or his guidance and help in completing this work successfully with good results. I would also like to express my gratitude to my Head of the Department Prof. Dr Piush Garg for his timely support and help.

\section{REFERENCES}

[1] Raj Mathews and Nidhi Chandra, Computer Mouse Tracking using Eye Tracking system based on Houghman Circle Detection Algorithm with Grid Analysis, Volume 40 No-13, International Journal of Computer Applications, February 2012.

[2] Pradipta Biswas, and Pat Langdon, A new system for disabled users involving eye gaze tracker and scanning system, Volume 5 No. 2 2011, Journal of Assistive Technologies.

[3] R.J.K. Jacob, Eye Movement-Based Human-Computer Interaction Techniques: Toward NonCommand Interfaces, in Advances in Human-Computer Interaction, Vol. 4, ed. by H.R. Hartson and D. Hix, pp. 151-190, Ablex Publishing Co., Norwood, N.J. (1993)

[4] Anjana Sharma and Pawanesh Abrol, Research issues in designing improved eye gaze based HCI techniques for Augmentative and Alternative Communication, International Journal of Emerging Technologies in Computational and Applied Sciences, 6(2), September-November, 2013, pp. 149153.

[5] Akhil Gupta, Akash Rathi and Dr. Y Radhika, "Hands-free PC Control" controlling of mouse cursor using eye movement, Internationla journal of Scientific and Research Publications, Volume 2, Issue 4, April 2012.

[6] Kohei Arai and Ronny Mardiyanto, Eyes based Electrical Wheel Chair Control System, International Journal of Advanced Computer Science and Applications, Vol. 2, No. 12, 2011.

[7] Robert J K Jacob, The use of Eye movements in Human-Computer Interaction Techniques: What you look at is what you get, ACM Transactions on Information Systems, Vol. 9, No. 3, April 1991, Pages 152-169.

[8] Yuan-Pin Lin, 1 Yi-Ping Chao, 2 Chung-Chih Lin, and 1 Jyh-Horng Chen, Webcam Mouse Using Face and Eye Tracking in Various Illumination Environments, Proceedings of the 2005 IEEE Engineering in Medicine and Biology 27th Annual Conference Shanghai, China, September 1-4, 2005.

[9] Web reference, Eye Controlled devices replace mouse, http://www.pcworld.com/article/47604/article.html.

[10] Web reference, Eye Tracking, Wikipedia Article, http://en.wikipedia.org/wiki/Eye_tracking.

[11] Alex Poole and Linden J. Ball, Eye tracking in Human-Computer Interaction and usability Research: Current Status and Future Prospects. Psychology Department, Lancastar University, UK

[12] Moniruzzaman Bhuiyan and Rich Picking, Gesture-controlled user interfaces, what have we done and what's next?, Center for Applied Internet Research, Glyndwr University, Wrexham, UK.

\section{AUTHORS}

Sagar Lakhmani is currently working for Accenture Services Private Limited. He has completed his graduation in Electrical and Electronics Engineering from Shri Ram Murti College of Engineering \& Technology, Bareilly, U.P., India in 2013. He has worked on various projects related to Robotics and image processing. He wishes to pursue further studies on these domains.

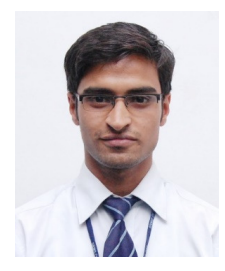

